\title{
Quantitative magnetic resonance imaging assessment of lateral atlantoaxial joint meniscoid composition: a validation study
}

Scott F Farrell1,2, Peter Stanwell ${ }^{3}$, Jon Cornwall ${ }^{4,5}$, Peter G Osmotherly ${ }^{3}$

1. RECOVER Injury Research Centre, NHMRC Centre for Research Excellence in Recovery Following Road Traffic Injuries, The University of Queensland, Brisbane, Australia

2. Menzies Health Institute Queensland, Griffith University, Gold Coast, Australia

3. School of Health Sciences, The University of Newcastle, Newcastle, Australia

4. Centre for Early Learning in Medicine, Otago Medical School, University of Otago, Dunedin, New Zealand

5. Institute for Health Sciences, Zurich University of Applied Sciences, Zurich, Switzerland

\section{Corresponding Author:}

Dr Scott Farrell

RECOVER Injury Research Centre, Level 7, Oral Health Building

The University of Queensland Herston Campus

Herston 4006 QLD

Australia

Email: scott.farrell@uq.edu.au Phone: +61 733464791 


\section{Abstract}

Purpose: Lateral atlantoaxial (LAA) joint meniscoid composition may have clinical significance in patients following neck trauma. However, the existing method of radiologically assessing meniscoid composition has an inherent element of subjectivity, which could contribute to measurement variability. The present study sought to investigate the accuracy of 2 point Dixon fat/water separation MRI as a quantitative assessment of LAA joint meniscoid composition.

Methods: Sixteen LAA joint meniscoids were excised from four cadavers (mean [SD] age 79.5 [3.7] years; one female) following cervical spine MRI (2-point Dixon, T1-weighted VIBE and T2-weighted SPACE sequences). Composition of LAA joint meniscoids was undertaken by i) histological examination by light microscopy, ii) calculation of fat fraction by Dixon MRI (both In-phase/Opposedphase and Fat/Water methods), and iii) the existing method of considering VIBE and SPACE signal intensities. Analysis was performed using the kappa statistic with linear weighting.

Results: Microscopy revealed three, five and eight meniscoids to be composed of adipose, fibroadipose and fibrous tissues, respectively. Dixon sequence MRI classified 11 of these meniscoids correctly, with 'substantial' level of agreement (In-phase/Opp-phase kappa statistic $=0.78[95 \% \mathrm{Cl}$ $0.38,1.17] ;$ Fat $/$ Water kappa statistic $=0.72[95 \% \mathrm{Cl} 0.32,1.11])$. Level of agreement between microscopy and the VIBE and SPACE method was 'poor' (kappa statistic $=0.02[95 \% \mathrm{Cl}-0.34,0.38]$ ). Conclusions: Findings suggest that Dixon fat/water separation MRI may have superior utility in the assessment of LAA joint meniscoid composition than the existing method of considering VIBE and SPACE signal intensities.

\section{Key Words}

Meniscoids, Synovial folds, Atlanto-axial joint, Magnetic resonance imaging, Histology, Cervical spine 


\section{Introduction}

Lateral atlantoaxial (LAA) joints contain folds of synovial membrane that protrude between the articular surfaces, referred to as meniscoids or synovial folds [1,2] (Fig. 1). These structures are suggested to improve the congruence and lubrication of joint structures [3], and their composition may have clinical significance. Histologically, meniscoids are lined with synovial membrane and are comprised of adipose or fibrous tissue, or a mixture of both [4].

Variation in meniscoid composition appears to be associated with chronic whiplash associated disorder [5], degenerative joint changes [4,6,7], and the presence of nerves [8]. Cervical spine meniscoid composition has been studied by dissection and light microscopy in cadavers $[3,4,6,7]$, and by magnetic resonance imaging (MRI) in vivo $[5,9]$. Categorisation of meniscoid composition by MRI was first described by Friedrich et al. [9], who evaluated meniscoid signal intensities on T1-weighted volumetric interpolated breath-hold examination (VIBE) and T2-weighted sampling perfection with amplification-optimised contrast using different angle evolutions (SPACE) sequences. These authors categorised meniscoids which were primarily hypointense on these sequences as 'mostly connective tissue' meniscoids (i.e. fibrous), primarily hyperintense on these sequences as 'mostly fatty' meniscoids (i.e. adipose), and partly hyper- and hypointense on these sequences as 'mixed tissue' meniscoids (i.e. fibroadipose).

However, this VIBE and SPACE method of assessing LAA meniscoid composition is arguably limited by a degree of inherent subjectivity, as determination of what is hyper- and hypointense signal could plausibly vary between raters. A strategy to address this limitation could be the use of quantitative assessment of adipose content of LAA meniscoids to guide evaluation of meniscoid composition, such as through the use of fat/water separation imaging sequences [10], allowing calculation of fat fraction of tissue within a region of interest (ROI). Quantitative calculation of fat content of musculoskeletal tissue has been undertaken in a number of contexts including the cervical spine [11] and leg [12]. However to date, no study has validated the use of fat/water separation imaging sequences to assess the composition of LAA joint meniscoids. 
The aim of this study was to evaluate the accuracy of quantitative assessment of LAA joint meniscoid composition using fat/water separation MRI. This was undertaken by examining level of agreement between quantitative assessment of meniscoid composition by fat/water separation MRI and the gold standard of histological assessment by microscopy. This was also compared with the level of agreement between the existing VIBE and SPACE method of assessment of meniscoid composition by MRI [9] and histological assessment by microscopy.

\section{Method}

A study employing MRI, dissection and histology was undertaken using cadaveric cervical spines, following approval by The University of Newcastle Human Research Ethics Committee (H-20150096). The study was undertaken in accordance with the 1964 Helsinki declaration and its later amendments. Written informed consent was attained from donors prior to death, and bodies were bequeathed to the anatomy department of The University of Newcastle, Australia in accordance with governing legislation.

Four cadavers were included in the study (mean [SD] age at death 79.5 [3.7] years; one female). Bodies were embalmed in a commercially available embalming fluid (Genelyn, Marden, Australia) mean (SD) 1.3 (1.3) days after death. The head and neck were removed from the trunk with a saw at approximately the T1-T2 level. This was performed with careful stabilisation, so that minimal traumatic force was transferred to the cervical spine. Each cervical spine and head subsequently underwent MRI.

\section{MRI Technique}

A Siemens Magnetom Skyra 3.0-Telsa unit (Siemens AG, Munich, Germany) with a 20-channel head and neck coil was used to perform the MRI scans. Each specimen (cervical spine and head) was contained in a waterproof plastic bag, positioned on a plastic tray in the scanner, and propped into a supine neutral cervical spine position. Images were acquired in the sagittal plane using T1-weighted VIBE, T2-weighted SPACE, and Dixon fat/water separation sequences (in-phase, opposed-phase, fat 
only, water only) (Table 1). LAA joint meniscoids are located at the ventral and dorsal aspects of the enclosing joints [3], and consequently the sagittal plane is most appropriate to visualise these structures.

Dissection

LAA joint meniscoids were then excised [8]. Overlying structures such as skin and muscles were removed using sharp and blunt dissection, in order to expose the vertebral column and skull, and allow disarticulation of the atlas from the occiput. Left and right articular pillars were separated from the vertebral bodies by cutting through the laminae and pedicles bilaterally with a band saw. Using a magnifying lamp to assist, dissection and disarticulation of each LAA joint was performed by slicing along the superior attachment of the joint capsule such that the superior joint surface could be gently lifted from the inferior surface, leaving the joint capsule and any enclosed meniscoids in situ. A scalpel was then used to excise each meniscoid and the attached joint capsule, for storage in a labelled specimen jar in $70 \%$ ethanol. Jar labels included details regarding cadaver identification number, side of body, and the position of the individual meniscoid within the joint.

\section{Histology}

Each LAA joint meniscoid was embedded in paraffin and sectioned at $4 \mu \mathrm{m}$ through the thickest part of its midline in the sagittal plane. Three contiguous sections from each meniscoid were then mounted on glass slides, stained with haematoxylin and eosin, and imaged with an Aperio AT2 Digital Pathology Scanner (Leica Biosystems Nussloch GmbH, Nussloch, Germany).

\section{Outcome Measures}

OsiriX v3.0.2 (Pixmeo, Geneva, Switzerland) radiological software was used to view MR images on a Dell P2210 1.76 megapixel monitor (Dell Inc., Round Rock, TX) in a quiet room with low lighting. MRI assessment was performed by a postdoctoral researcher (SF) with three years' experience researching cervical spine meniscoid morphology using MRI, including receiving prior training from a 
senior radiologist. MRI evaluation of LAA joint meniscoid composition was performed prior to viewing the microscope slides, to prevent prior knowledge of microscopy classifications influencing MRI interpretation (i.e. blinded assessment).

Each LAA joint meniscoid was first classified using the Friedrich et al. [9] VIBE and SPACE method: primarily hyperintense on these sequences were 'mostly fatty' meniscoids (i.e. adipose), primarily hypointense on these sequences were 'mostly connective tissue' meniscoids (i.e. fibrous), and partly hyper- and hypointense on these sequences were 'mixed tissue' meniscoids (i.e. fibroadipose).

Classification of each LAA joint meniscoid was performed a second time two weeks later for testing of intra rater reliability.

Quantitative MRI assessment of the fat fraction of each LAA joint meniscoid was then performed using the set of four Dixon sequences (in-phase, opposed-phase, fat only, water only). This involved manually tracing a ROI around the sagittal plane cross-section of each LAA joint meniscoid on the inphase sequence. The identical ROI was then transposed to the same slice of the opposed-phase, fat only and water only sequences (Figure 2). Mean signal intensity (MSI) for the ROI was then recorded for the four sequences. Fat fraction was calculated using MSI from the in-phase and opposed-phase sequences, referred to from here as 'Dixon In/Opp':

$$
\frac{\text { in phase MSI - opposed phase MSI }}{2 \times \text { in phase MSI }}
$$

Fat fraction was also calculated using MSI from the fat only and water only sequences, referred to from here as Dixon Fat/Water:

$$
\frac{\text { fat only MSI }}{\text { fat only MSI }+ \text { water only } M S I}
$$

This was repeated on five more contiguous slices (to make six in total) through the thickest part of the middle of each structure, to allow calculation of mean fat fraction for each meniscoid. Calculation of meniscoid mean fat fraction was performed a second time for all specimens one week later for testing 
of intra rater reliability.

Histological assessment of LAA joint meniscoid composition by light microscopy was undertaken using Aperio eSlide Manager browser-based viewing platform (Leica Biosystems Nussloch GmbH, Nussloch, Germany). This was done by a postdoctoral researcher (SF) with five years' experience performing histological research on cervical spine meniscoids. Each LAA joint meniscoid was classified in accordance with previous microscopy studies [4]: meniscoids primarily composed of adipocytes were classified as 'adipose type', meniscoids primarily comprising fibrous tissue were classified as 'fibrous type', and those comprising a mixture of adipose and fibrous tissues were classified as 'fibroadipose type'. Classification of each LAA joint meniscoid by microscopy was performed a second time two weeks later to allow testing of intra rater reliability.

\section{Statistical Analysis}

Analysis was performed using Stata 13.1 (StataCorp, College Station, TX). Data for MRI (VIBE and SPACE method, Dixon In/Opp, Dixon Fat/Water) and microscopy classifications were initially analysed descriptively. Levels of agreement for classification of LAA joint meniscoid composition between the MRI methods (VIBE and SPACE method, Dixon In/Opp, Dixon Fat/Water) as compared to microscopy classifications were calculated using the kappa statistic with linear weighting, with calculation of $95 \%$ confidence intervals (Cls) [13]. Kappa was interpreted according to the guidelines proposed by Landis and Koch [14]. Intra rater reliability of the VIBE and SPACE method and microscopy assessment of meniscoid composition was also examined using the kappa statistic with linear weighting. Intra rater reliability of fat fraction was calculated using two-way mixed-effects intraclass correlation coefficients (ICC [3,k]), and interpreted as per Fleiss [15].

\section{Results}

All eight LAA joints of the four cadavers were successfully disarticulated. Each LAA joint contained a ventral and a dorsal meniscoid, providing 16 meniscoids for inclusion in the study (Figure 3). 
VIBE and SPACE Assessment of Meniscoid Composition

Using the Friedrich et al. [9] VIBE and SPACE method, two LAA joint meniscoids were classified as adipose in composition (both dorsal meniscoids), nine were classified as fibroadipose in composition (six ventral, three dorsal), and five were classified as fibrous in composition (two ventral, three dorsal). Intra rater reliability for this method of MRI assessment of meniscoid composition was 'moderate' [14]: kappa statistic $=0.52(95 \% \mathrm{Cl} 0.14,0.89)$.

\section{Quantitative Assessment of Fat Fraction for Meniscoid Composition}

Median (IQR) [range] for fat fraction calculated by Dixon In/Opp was $0.17(0.08,0.25)[0.05,0.33]$. Median (IQR) [range] for fat fraction calculated by Dixon Fat/Water was $0.19(0.12,0.26)[0.07,0.41]$. Intra rater reliability of fat fraction was 'excellent' [15], with ICC $(3,6)=0.98(95 \% \mathrm{Cl} 0.95,0.99)$ for Dixon In/Opp and ICC $(3,6)=0.98(95 \% \mathrm{Cl} 0.94,0.99)$ for Dixon Fat/Water.

\section{Microscopy Assessment of Meniscoid Composition}

Using microscopy, three LAA joint meniscoids were classified as adipose in composition (all ventral), five were classified as fibroadipose (all ventral), and eight were classified as fibrous (all dorsal) (Figure 4). Intra rater reliability for this method of assessment of meniscoid composition was 'almost perfect' [14]: kappa statistic $=0.84(95 \% \mathrm{Cl} 0.47,1.22)$.

\section{Comparison of VIBE and SPACE Assessment of Composition to Microscopy}

Comparison of assessment of cervical spine meniscoid composition using the VIBE and SPACE method described by Friedrich et al. [9] with assessment by microscopy can be seen in Table 2. Level of agreement on meniscoid composition for the VIBE and SPACE MRI method compared with microscopy was 'slight' [14]: kappa statistic $=0.02(95 \% \mathrm{Cl}-0.34,0.38)$. 
Median (IQR) [range] fat fraction for Dixon In/Opp and Dixon Fat/Water presented by meniscoid composition on microscopy can be seen in Table 3. Based on these data, the following fat fraction cut offs were selected for categorising fat fraction into the three meniscoid compositions: $\leq 0.180$ fibrous meniscoid; 0.181 to 0.241 fibroadipose meniscoid; $\geq 0.242$ adipose meniscoid. Using these fat fraction cut offs, four meniscoids were classified as adipose, four as fibroadipose, and eight as fibrous using the Dixon In/Opp method. Using the Dixon Fat/Water method, five meniscoids were classified as adipose, three as fibroadipose, and eight as fibrous. Agreement between Dixon In/Opp assessment of meniscoid composition by MRI and assessment by microscopy can be seen in Table 4. Level of agreement was 'substantial' [14]: kappa statistic $=0.78(95 \% \mathrm{Cl} 0.38,1.17)$. Agreement between Dixon Fat/Water assessment of meniscoid composition by MRI and assessment by microscopy can be seen in Table 5. Level of agreement was 'substantial' [14]: kappa statistic $=0.72$ $(95 \% \mathrm{Cl} 0.32,1.11)$.

\section{Discussion}

This is the first study to evaluate assessment of LAA joint meniscoid composition using quantitative measurement of Dixon fat/water separation MRI. Findings indicate that level of agreement between both the Dixon In/Opp and Dixon Fat/Water approaches and the gold standard of microscopy were 'substantial', considerably higher than the 'slight' agreement observed in this instance between the previously described VIBE and SPACE method [9] and microscopy. These results are comparable to those reported by Smith et al. [16], who examined muscle fat fraction in pig and rabbit tissue using Dixon fat/water separation MRI as compared with histological examination by microscopy. Similar to the present study, Smith et al. found that measurement of muscle fat fraction by MRI was consistent with that measured by microscopy.

At present, the clinical significance of LAA joint meniscoids is not clear. As they may contain blood vessels and nerves, it has been suggested that damage to these structures in trauma, such as a whiplash injury, may have clinical implications as a source of nociceptive input following injury $[2,8,17,18]$. Biomechanical evidence indicates that LAA joints are potentially subject to hyperflexion in a whiplash event in a manner that may exceed physiological limits of the tissue [19]. Further, autopsy studies reveal bruising of LAA meniscoids in victims of fatal road traffic crashes, suggesting that these 
structures are vulnerable to damage in trauma [20]. It is plausible that injury to LAA joint meniscoids could lead to alterations in morphology, such as through chronic inflammation [21] and/or decreased mobility [22] leading to scar tissue formation or fibrosis [17]. As such, capacity to effectively assess composition of LAA joint meniscoids has value in a research setting, with scope for future potential clinical utility if applicable.

\section{Limitations}

The cadavers included in this study were embalmed. Although embalmed cadavers are often utilised in studies comparing MRI with morphology [23], the embalming process can affect MR image quality through altered water mobility and signal intensity, as well as fluid accumulation in joint spaces and soft tissue swelling $[24,25]$. These processes could plausibly influence MRI assessment of meniscoid composition through the Friedrich et al. [9] VIBE and SPACE method, as well as measurement of signal intensity on the Dixon fat/water separation sequences.

\section{Future Research Directions}

This investigation provides proof of concept for the use of quantitative measurement of Dixon fat/water separation MRI in the assessment of LAA joint meniscoid composition. As such, the cut off parameters for fat fraction values to define meniscoid composition classifications in this study were determined post hoc. Given that the embalming process may alter MRI signal intensity, it is possible that parameters for fat fraction for classification of meniscoids in living humans may differ from those used in the present study. Future research should refine fat fraction parameters for classifications of meniscoid composition, ideally in living tissue.

\section{Conclusions}

The present study provides preliminary evidence for utility of Dixon fat/water separation MRI in assessment of LAA joint meniscoid composition. Agreement between this assessment technique and 
the gold standard of microscopy was 'substantial', however as this work was undertaken in embalmed cadavers, further investigation is required to determine reference values in living tissue. 


\section{References}

[1] Webb AL, Darekar A, Rassoulian H. The influence of age, anthropometrics and range of motion on the morphometry of the synovial folds of the lateral atlanto-axial joints: a pilot study. Eur Spine J, $2011,20(4): 542-549$

[2] Webb AL, Collins P, Rassoulian H, Mitchell BS. Synovial folds - A pain in the neck? Manual Therapy, 2011,16:118-124.

[3] Mercer S, Bogduk N. Intra-articular inclusions of the cervical synovial joints. Journal of Rheumatology, 1993,32 (8):705-710.

[4] Farrell SF, Osmotherly PG, Cornwall J, Rivett DA. Morphology and morphometry of lateral atlantoaxial joint meniscoids. Anatomical Science International, 2016,91:89-96. doi:10.1007/s12565015-0276-z.

[5] Farrell SF, Osmotherly PG, Cornwall J, Lau P, Rivett DA. Morphology of cervical spine meniscoids in individuals with chronic whiplash associated disorder: a case-control study. Journal of Orthopaedic and Sports Physical Therapy, 2016,46 (10):902-910. doi:10.2519/jospt.2016.6702.

[6] Inami S, Kaneoka K, Hayashi K, Ochiai N. Types of synovial fold in the cervical facet joint. Journal of Orthopaedic Science, 2000,5:475-480.

[7] Farrell SF, Osmotherly PG, Cornwall J, Rivett DA. The anatomy and morphometry of cervical zygapophyseal joint meniscoids. Surg Radiol Anat, 2015,37 (7):799-807. doi:10.1007/s00276-0141406-3.

[8] Farrell SF, Osmotherly PG, Cornwall J, Rivett DA. Immunohistochemical investigation of nerve fibre presence and morphology in elderly cervical spine meniscoids. The Spine Journal, 2016,16 (10):1244-1252. doi:10.1016/j.spinee.2016.06.004.

[9] Friedrich KM, Reiter G, Pretterkliedber ML, Pinker K, Friedrich M, Trattnig S, Salomonowitz E. Reference data for In Vivo magnetic resonance imaging properties of meniscoids in the cervical zygapophyseal joints. Spine, 2008,33 (21):E778-E783.

[10] Elliott JM, Walton DW, Rademaker A, Parrish TB. Quantification of cervical spine muscle fat: a comparison between T1-weighted and multi-echo gradient echo imaging using a variable projection algorithm (VAPRO). BMC Medical Imaging, 2013,13 (30). 
[11] Elliott JM, Courtney D, Rademaker A, Pinto D, Sterling M, Parrish TB. The rapid and progressive degeneration of the cervical multifidus in whiplash: a MRI study of fatty infiltration. Spine, 2015,40 (12):E694-E700.

[12] Alizai H, Nardo L, Karampinos D, Joseph G, Yap S, Baum T, Krug R, Majumdar S, Link T. Comparison of clinical semi-quantitative assessment of muscle fat infiltration with quantitative assessment using chemical shift-based water/fat separation in MR studies of the calf of postmenopausal women. European Radiology, 2012,22 (7):1592-1600.

[13] Sim J, Wright C. The kappa statistic in reliability studies: use, interpretation and sample size requirements. Physical Therapy, 2005,85 (3):257-268.

[14] Landis JR, Koch GG. The measurement of observer agreement for categorical data. Biometrics, 1977,33 (1):159-174.

[15] Fleiss JL. The Design and Analysis of Clinical Experiments. John Wiley \& Sons, New York, 1986, doi:

[16] Smith AC, Parrish TB, Abbott R, Hoggarth MA, Mendoza K, Chen YF, Elliott JM. Muscle-fat MRI: 1.5 Tesla and 3.0 Tesla versus histology. Muscle \& Nerve, 2014,50:170-176.

[17] Farrell SF, Osmotherly PG, Cornwall J, Sterling M, Rivett DA. Cervical spine meniscoids: an update on their morphological characteristics and potential clinical significance. Eur Spine J, 2017,26 (4):939-947. doi:10.1007/s00586-016-4915-4.

[18] Farrell SF, Khan S, Osmotherly PG, Sterling M, Cornwall J, Rivett DA. Lateral atlantoaxial joint meniscoid volume in individuals with whiplash associated disorder: a case-control study. Musculoskeletal Science and Practice, 2018,33:46-52.

[19] Grauer JN, Panjabi M, Cholewicki J, Nibu K, Dvorak J. Whiplash produces an S-shaped curvature of the neck with hyperextension at lower levels. Spine, 1997,22 (21):2489-2494.

[20] Uhrenholt L, Grunnet-Nilsson N, Hartvigsen J. Cervical spine lesions after road traffic accidents: a systematic review. Spine, 2002,27 (17):1934-1941.

[21] Barr A, Barbe M, Clark B. Work-related musculoskeletal disorders of the hand and wrist: epidemiology, pathophysiology, and sensorimotor changes. Journal of Orthopaedic and Sports Physical Therapy, 2004,34 (10):610-627.

[22] Cramer GD, Henderson CN, Little JW, Daley C, Grieve TJ. Zygapophyseal joint adhesions after induced hypomobility. Journal of Manipulative and Physiological Therapeutics, 2010,33 (7):508-518. 
[23] Gregg J, Silberstein M, Schneider T, Kerr JB, Marks P. Sonography of plantar plates in cadavers: correlation with MRI and histology. American Journal of Roentgenology, 2006,186:948-955.

[24] Schramek GGR, Stoevesandt D, Reising A, Kielstein JT, Hiss M, Kielstein H. Imaging in anatomy: a comparison of imaging techniques in embalmed human cadavers. BMC Medical Education, 2013,13 (143).

[25] van der Made AD, Maas M, Beenen LFM, Oostra RJ, Kerkhoffs GMMJ. Postmortem imaging exposed: an aid in MR imaging of musculoskeletal structures. Skeletal Radiology, 2013,42:467-472. doi:10.1007/s00256-012-1515-1. 


\section{Figures and Legends}

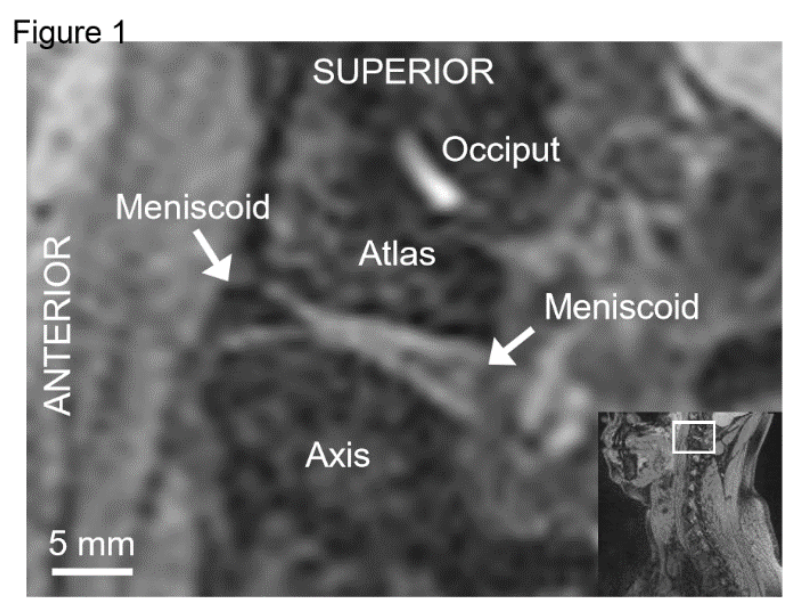

Fig. 1 Sagittal magnetic resonance image through a right lateral atlantoaxial joint demonstrating ventral and dorsal meniscoids, using a double-echo steady state sequence in vivo in a 26-year-old female 
Figure 2
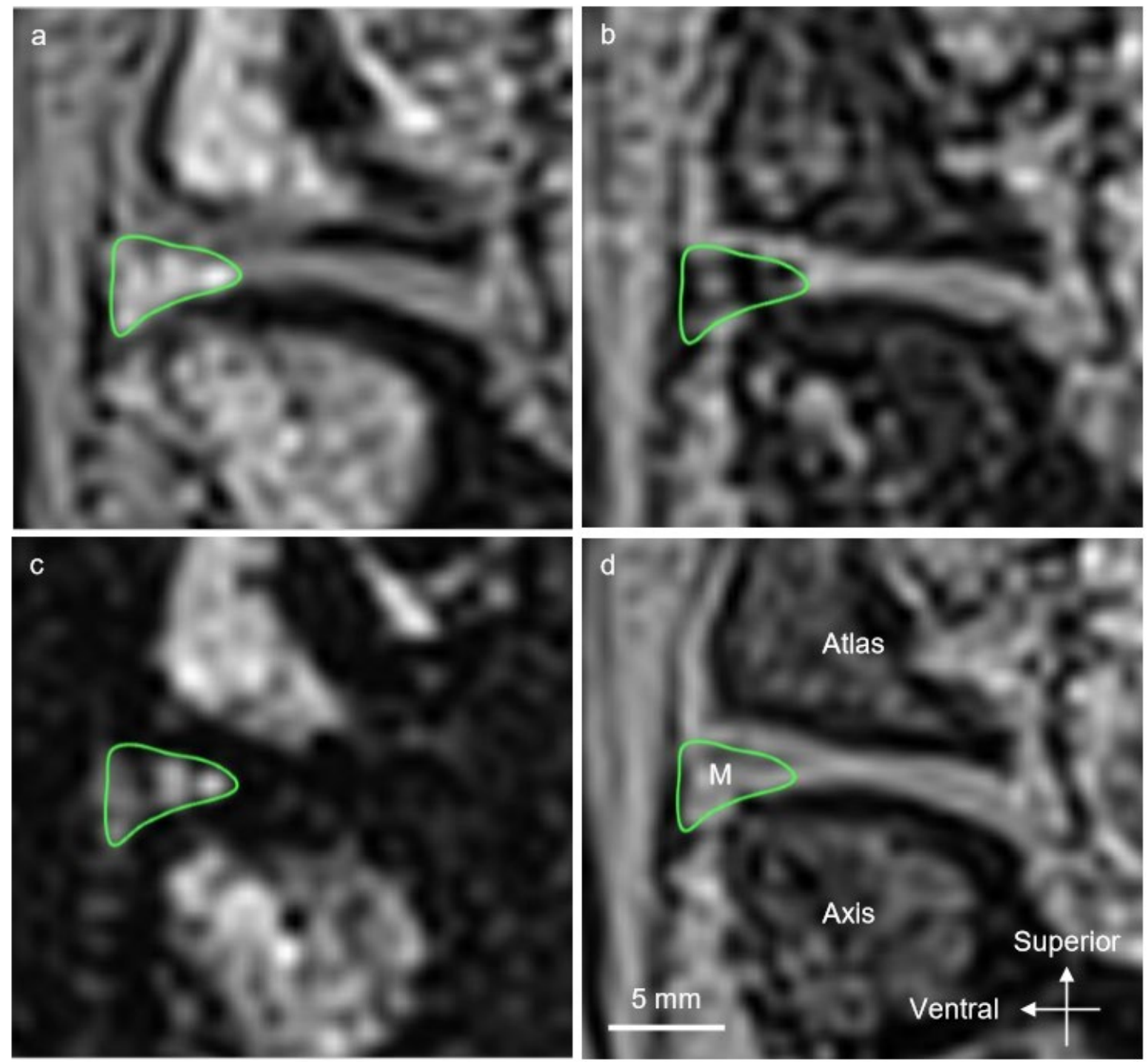

Fig. 2: Sagittal plane Dixon sequence magnetic resonance images, with green region of interest delineating meniscoid (M) at ventral aspect of lateral atlantoaxial joint. (a) In-phase image, (b) opposed-phase image, (c) fat only image, (d) water only image 


\section{Figure 3}

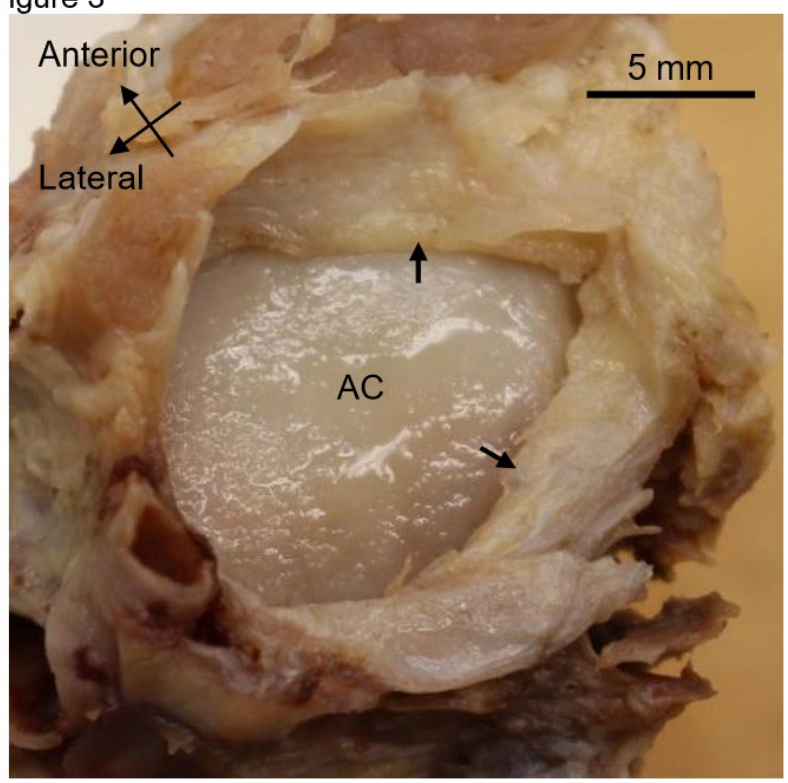

Fig 3: Superior view of disarticulated left lateral atlantoaxial joint, containing meniscoids (black arrows) at ventral and dorsal aspects of joint. $A C=$ articular cartilage 


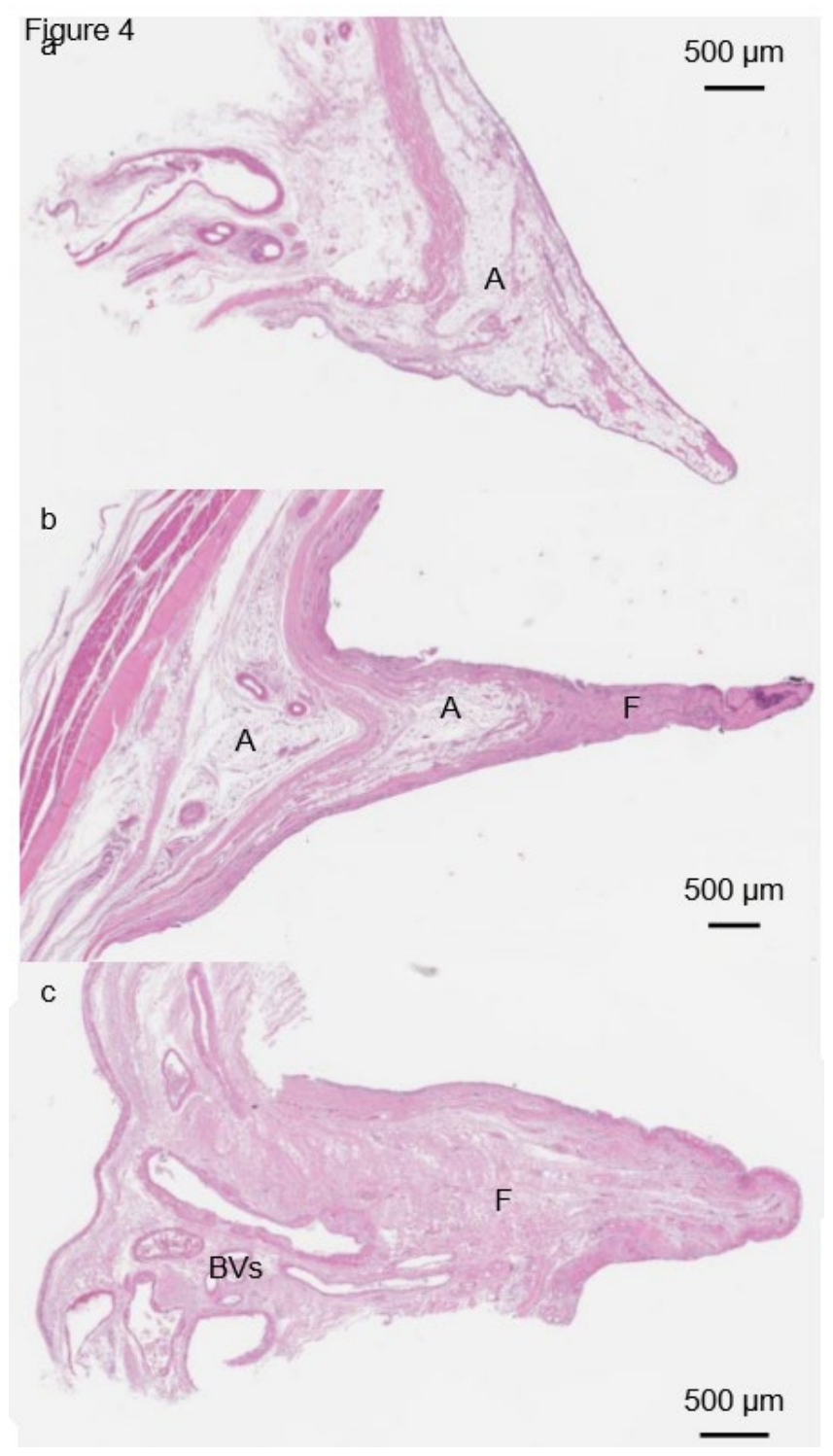

Fig. 4: Sagittal sections of lateral atlantoaxial joint meniscoids, demonstrating histological classifications (light microscopy, haematoxylin and eosin). (a) Adipose meniscoid removed from the ventral aspect of a joint. (b) Fibroadipose meniscoid removed from the ventral aspect joint. (c) Fibrous meniscoid removed from the dorsal aspect of a joint. A: adipose tissue; BVs: blood vessels; F: fibrous tissue 
Tables

Table 1: Details of magnetic resonance imaging sequences. BW = bandwidth; FOV = field of view; SPACE = sampling perfection with amplification-optimised contrast using different angle evolutions; TE = echo time; TR = repetition time; TSE = turbo spin echo; VIBE = volumetric interpolated breathhold examination

\begin{tabular}{|l|l|l|l|}
\hline Parameter & T1-weighted VIBE & T2-weighted SPACE & Dixon TSE Sequences \\
\hline Acquisition time & $12 \mathrm{~min} 38 \mathrm{~s}$ & $14 \mathrm{~min} 41 \mathrm{~s}$ & $10 \mathrm{~min} 33 \mathrm{~s}$ \\
\hline FOV & $200 \times 200 \mathrm{~mm}^{2}$ & $250 \times 250 \mathrm{~mm}^{2}$ & $289 \times 289 \mathrm{~mm}^{2}$ \\
\hline Matrix & $320 \times 288$ & $301 \times 320$ & $448 \times 448$ \\
\hline Slice thickness & $0.6 \mathrm{~mm}$ & $0.6 \mathrm{~mm}$ & $0.7 \mathrm{~mm}$ \\
\hline Spatial resolution & $0.6 \times 0.6 \times 0.6 \mathrm{~mm}^{2}$ & $0.8 \times 0.8 \times 0.6 \mathrm{~mm}^{2}$ & $0.7 \times 0.7 \times 0.7 \mathrm{~mm}^{2}$ \\
\hline TE & $3.38 \mathrm{~ms}$ & $136 \mathrm{~ms}$ & $14 \mathrm{~ms}$ \\
\hline TR & $10.80 \mathrm{~ms}$ & $1500 \mathrm{~ms}$ & $711 \mathrm{~ms}$ \\
\hline Flip angle & $10^{\circ}$ & $115^{\circ}$ & $160^{\circ}$ \\
\hline No. of averages & 3 & 2 & 1 \\
\hline BW & $240 \mathrm{~Hz} /$ pixel & $625 \mathrm{~Hz} /$ pixel & $385 \mathrm{~Hz} /$ pixel \\
\hline
\end{tabular}


Table 2: Kappa table showing agreement between classifications of lateral atlantoaxial joint meniscoid composition by Friedrich et al. [9] VIBE and SPACE method with classification by microscopy (gold standard). Diagonal cells (highlighted grey) show the number of instances of agreement on classification of individual meniscoids by MRI and microscopy, e.g. 4/5 fibroadipose meniscoids were correctly classified. The unshaded cells show the number of individual meniscoids that the classification techniques disagreed upon, e.g. 2 meniscoids that were classified as fibrous type on microscopy were incorrectly classified as adipose on MRI

\begin{tabular}{|c|c|c|c|c|c|}
\hline & \multicolumn{4}{|c|}{ VIBE and SPACE MRI Assessment } \\
\hline & & $\begin{array}{c}\text { 'Adipose } \\
\text { type' }\end{array}$ & $\begin{array}{c}\text { 'Fibroadipose } \\
\text { type' }\end{array}$ & $\begin{array}{c}\text { 'Fibrous } \\
\text { type' }\end{array}$ & Total \\
\hline \multirow{4}{*}{$\begin{array}{l}\text { Microscopy } \\
\text { Assessment }\end{array}$} & 'Adipose type' & 0 & 2 & 1 & 3 \\
\hline & $\begin{array}{l}\text { 'Fibroadipose } \\
\text { type' }\end{array}$ & 0 & 4 & 1 & 5 \\
\hline & 'Fibrous type' & 2 & 3 & 3 & 8 \\
\hline & Total & 2 & 9 & 5 & 16 \\
\hline
\end{tabular}


Table 3: Lateral atlantoaxial joint meniscoid fat fraction, calculated by Dixon In/Opp and Dixon Fat/Water quantitative MRI approaches, split by meniscoid composition on microscopy

\begin{tabular}{|l|l|l|l|l|}
\hline \multicolumn{2}{|c|}{} & \multicolumn{3}{|c|}{ 'Ficroscopy Assessment } \\
\cline { 3 - 5 } & \multicolumn{2}{|c|}{$\begin{array}{l}\text { 'Adipose Type' } \\
(\mathbf{n}=\mathbf{3})\end{array}$} & $\begin{array}{l}\text { 'Fibroadipose } \\
\text { Type' (n = 5) }\end{array}$ & $\begin{array}{l}\text { 'Fibrous Type' } \\
\text { (n = 8) }\end{array}$ \\
\hline $\begin{array}{l}\text { Fat fraction } \\
\text { median (IQR) }\end{array}$ & Dixon In/Opp & $0.25(0.19,0.33)$ & $0.24(0.24,0.25)$ & $0.08(0.05,0.10)$ \\
[range] & {$[0.19,0.33]$} & {$[0.19,0.30]$} & {$[0.05,0.15]$} \\
\cline { 2 - 5 } & Dixon Fat/Water & $0.27(0.19,0.41)$ & $0.25(0.24,0.28)$ & $0.12(0.09,0.17)$ \\
& & {$[0.19,0.41]$} & {$[0.22,0.31]$} & {$[0.07,0.18]$} \\
\hline
\end{tabular}


Table 4: Kappa table showing agreement between classifications of lateral atlantoaxial joint meniscoid composition by Dixon In/Opp quantitative MRI assessment with classification by microscopy (gold standard). Diagonal cells (highlighted grey) show the number of instances of agreement on classification of individual meniscoids by MRI and microscopy

\begin{tabular}{|c|l|l|l|l|l|}
\hline \multicolumn{2}{|c|}{} & \multicolumn{3}{c|}{ Classification Based on In/Opp Fat Fraction } \\
\cline { 3 - 7 } & $\begin{array}{c}\text { 'Adipose } \\
\text { type' }\end{array}$ & $\begin{array}{c}\text { 'Fibroadipose } \\
\text { type' }\end{array}$ & $\begin{array}{c}\text { 'Fibrous } \\
\text { type' }\end{array}$ & Total \\
\hline \multirow{4}{*}{$\begin{array}{c}\text { Microscopy } \\
\text { Assessment }\end{array}$} & $\begin{array}{l}\text { 'Adipose type' } \\
\text { 'Fibroadipose }\end{array}$ & 2 & 1 & 0 & 3 \\
\cline { 2 - 7 } & type' & & 3 & 0 & 5 \\
\cline { 2 - 7 } & 'Fibrous type' & 0 & 0 & 8 & 16 \\
\cline { 2 - 7 } & Total & 4 & 4 & 8 & 8 \\
\hline
\end{tabular}


Table 5: Kappa table showing agreement between classifications of lateral atlantoaxial joint meniscoid composition by Dixon Fat/Water quantitative MRI assessment with classification by microscopy (gold standard). Diagonal cells (highlighted grey) show the number of instances of agreement on classification of individual meniscoids by MRI and microscopy

\begin{tabular}{|c|l|l|l|l|l|}
\hline \multicolumn{2}{|c|}{} & \multicolumn{3}{c|}{ Classification Based on Fat/Water Fat Fraction } \\
\cline { 3 - 7 } & $\begin{array}{c}\text { 'Adipose } \\
\text { type' }\end{array}$ & $\begin{array}{c}\text { 'Fibroadipose } \\
\text { type' }\end{array}$ & $\begin{array}{c}\text { 'Fibrous } \\
\text { type' }\end{array}$ & Total \\
\hline \multirow{4}{*}{$\begin{array}{c}\text { Microscopy } \\
\text { Assessment }\end{array}$} & $\begin{array}{l}\text { 'Adipose type' } \\
\text { 'Fibroadipose }\end{array}$ & 2 & 1 & 0 & 3 \\
\cline { 2 - 7 } & type' & & 2 & 0 & 5 \\
\cline { 2 - 7 } & 'Fibrous type' & 0 & 0 & 8 & 8 \\
\cline { 2 - 7 } & Total & 5 & 3 & 8 & 16 \\
\hline
\end{tabular}

